\title{
Do we have to reduce the recall period? Validity of a daily physical activity questionnaire (PAQ24) in young active adults
}

\author{
B. Novak ${ }^{1 \dagger}$, P. Holler ${ }^{1,2 \dagger}$, J. Jaunig ${ }^{1}$, W. Ruf ${ }^{1,3}$, M. N. M. van Poppel ${ }^{1}$ and M. C. Sattler ${ }^{1 *}$
}

\begin{abstract}
Background: Combining the strengths of physical activity (PA) diaries and questionnaires may be needed to improve the unsatisfying measurement quality of existing PA questionnaires. This study investigated the construct validity of a short PA questionnaire (Physical Activity Questionnaire for $24 \mathrm{~h}$ [PAQ24]) with a recall period of one day.

Methods: In this cross-sectional study, participants completed the PAQ24 on seven consecutive days while wearing an accelerometer (GENEActiv). Thereafter, the Global Physical Activity Questionnaire (GPAQ) was completed. Spearman correlation coefficients and Bland-Altman analysis were used to assess construct validity.

Results: Overall, 50 active adults ( 11 women, mean age $=25.1 \pm 2.5$ ) participated. Relative agreements between Total PA of PAQ24 and accelerometer were $0.37 \leq \rho \leq 0.72$ for each day with satisfying agreement on five out of seven days. Weekly relative agreement for Total PA was moderate $(\rho=0.44)$. Relative agreements between PAQ24 and GPAQ were $\rho=0.43$ for Total PA. Daily and weekly absolute agreements were poor indicated by wide limits of agreement.

Conclusions: In contrast to weekly Total PA, the majority of daily results of the PAQ24 showed satisfying construct validity. A short recall period may improve the measurement quality of PA questionnaires, but measurement errors and the costs of multiple administrations must be considered in future studies.
\end{abstract}

Keywords: Physical activity, Questionnaire, Self-report, Measurement, Validity

\section{Background}

Physical activity (PA) has been linked to a great number of physical and mental health benefits [1]. For example, there is strong evidence that moderate-to-vigorous physical activity (MVPA) reduces the risks of type 2 diabetes, coronary heart disease, depression and all-cause mortality $[2,3]$. To achieve substantial health benefits, adults should perform at least 150 min of moderate-to-vigorous intensity aerobic activities per week as well as musclestrengthening activities on two or more days per week [4]. This evidence is often based upon large cohort studies or

\footnotetext{
* Correspondence: matteo.sattler@uni-graz.at

${ }^{\dagger}$ B. Novak and P. Holler contributed equally to this work.

'Institute of Sport Science, University of Graz, Graz, Austria

Full list of author information is available at the end of the article
}

randomized controlled trials using self-reported PA. However, the conclusions drawn from these studies depend on the quality of the assessment of exposures and outcomes.

For a long time, PA was exclusively assessed by selfreport measures (e.g., questionnaires, diaries) due to the lack of alternatives. Today, research can rely on devicebased measures such as accelerometers. Since accelerometry is not always feasible in large epidemiological studies and because questionnaires still provide important information about type (e.g., walking, cycling) and domains of PA (e.g., home, leisure time), questionnaires remain popular to gather valuable information at low cost [5]. In fact, a large part of the evidence forming the basis of current PA guidelines is based on questionnaire data [1]. Until now, many questionnaires have been

(c) The Author(s). 2020 Open Access This article is distributed under the terms of the Creative Commons Attribution 4.0 International License (http://creativecommons.org/licenses/by/4.0/), which permits unrestricted use, distribution, and reproduction in any medium, provided you give appropriate credit to the original author(s) and the source, provide a link to the Creative Commons license, and indicate if changes were made. The Creative Commons Public Domain Dedication waiver (http://creativecommons.org/publicdomain/zero/1.0/) applies to the data made available in this article, unless otherwise stated. 
developed since no gold standard for the measurement of PA exists [6].

Despite the existence of many different questionnaires, no conclusive recommendations can be provided for the best questionnaires to assess PA in various populations due to the inconsistent results regarding their measurement properties $[7,8]$. The results for construct validity are often unsatisfying. Average correlations with accelerometer data of $r=0.22$ for moderate and $r=0.32$ for vigorous PA were reported [8], and for total PA the coefficients ranged from 0.04 to 0.47 [9]. This means that there is at best only $25 \%$ shared variance between these two methods [10]. Moreover, it seems that over the last decades the measurement quality of PA questionnaires did not considerably improve, for example, when comparing newly developed with already existing versions [11].

One shortcoming of a PA questionnaire is the reporting error associated with the recall period [12]. In a typical administration, a person is asked to recall and summarize all physical activities performed in a defined period (e.g., the past/usual week or past month). This means that a person should be able to correctly report frequency, duration and intensity of PA over the defined period. However, the person's PA level is determined not only by the true amount of PA but also by the ability to recall all relevant activities. The accuracy of the recall may further be influenced by the type of activity. For example, the recall may be more difficult for sporadic, brief and low-intensity activities [13, 14].

However, evidence is accumulating that also light PA provides important health benefits such as reductions in mortality risks and improved cardiometabolic health, especially for inactive populations [15-17]. Moreover, current PA guidelines emphasized that also incidental, intermittent activities (e.g., less than 10 consecutive minutes) provide health benefits $[1,4]$. Many existing questionnaires do not capture these brief activities [8]. A short recall period may be needed to capture all these relevant activities and, thus, help to improve the quality of PA measurement using questionnaires. The advantages of a short recall period have been highlighted previously. For example, Matthews et al. [14] acknowledged that it will reduce the cognitive demands of the participants because the recall would strongly rely on the recollection of behaviors using episodic memories. Hence, the authors recommended using multiple short-term recalls to obtain more accurate behavior-disease associations.

Although shorter recall periods (e.g., previous day) provide the potential to limit reporting errors, they were typically only applied in diaries or records such as within the "Activities Completed Over Time in 24 Hours" (ACT24) [18]. These formats tend to show better agreement with device-based measures of PA (e.g., $0.48 \leq r \leq 0.60$ for
ACT24) but at the expense of high burden for participants. Therefore, feasibility on a weekly basis, for example using multiple measurements, in large studies is limited. Until now, only two PA questionnaires used a recall period of one day and both showed good agreements with accelerometers (e.g., $r=0.71$ for the Danish Physical Activity Questionnaire and $r=0.74$ for the Daily Activity Questionnaire) [19, 20].

Since these two questionnaires are either too long or developed for a population of patients (i.e., after total hip arthroplasty), the measurement properties of a short daily PA questionnaire in a non-patient population are unknown. A short version could also help to increase feasibility when using multiple measurements. Therefore, the aim of this study was to assess the construct validity of a short self-administered daily PA questionnaire (Physical Activity Questionnaire for 24 h [PAQ24]) within a sample of the healthy population, namely young active adults. For the design of the questionnaire, we modified the International Physical Activity Questionnaire - Short Form (IPAQ-SF) and reduced the recall period from one week to one day.

Regarding PA, we hypothesized that the use of a daily recall period would: i) result in satisfying relative agreement between PAQ24 and other measures of the construct (established PA questionnaire, accelerometer). As recommended [8], we assumed correlations $\geq 0.50$ between questionnaire and accelerometer and $\geq 0.70$ between PA questionnaires as evidence for satisfying relative agreement; ii) result in satisfying absolute agreement between PAQ24 and these instruments. No thresholds for absolute agreement could be defined since there is no gold standard for the measurement of PA [6].

\section{Methods}

\section{Study design and setting}

In this cross-sectional study, recruitment of participants started on March 3, 2018 and was conducted at the Institute of Sport Science of the University of Graz, Austria. Participants of three different university courses were asked to participate in the study. Participants received no compensation but were able to request their individual results. Data collection was between May 4 and June 14, 2018. The initial assessments included anthropometric (measured weight and height, used to calculated bodymass-index [BMI, $\left.\mathrm{kg} / \mathrm{m}^{2}\right]$ ), sociodemographic data and individual aerobic capacity using the Chester Step Test [21]. This valid submaximal test was used to estimate maximum oxygen consumption $\left(\mathrm{VO}_{2 \max }[\mathrm{ml} / \mathrm{kg} / \mathrm{min}]\right)$ and to describe the fitness level of the sample [22]. Participants completed the paper-pencil version of the PAQ24 at the end of each day for seven consecutive days and wore an accelerometer for the same period. In the second week, participants completed the Global Physical Activity 
Questionnaire (GPAQ) and a questionnaire obtaining participants' experiences with the PAQ24 (i.e., What were your experiences with the questionnaire? Were there any difficulties when completing the questionnaire? What would you like to change, remove or add to the questionnaire?).

\section{Participants}

Participants were students at the Institute of Sport Science and: (i) were registered for the programs sport science and/or physical education; (ii) had no self-reported acute physical injury; (iii) were fluent in the German language; and (iv) aged $\geq 18$ years.

\section{Measurements}

\section{PAQ24}

The IPAQ-SF - a validated questionnaire to assess PA in adults with reference to activities of the past or usual week [23] - was used to design our daily PA questionnaire (PAQ24). The PAQ24 aims to capture the total volume of PA (i.e., MVPA including walking) during the waking hours of a single day. Participants were instructed to complete the questionnaire before going to bed on seven consecutive days. As a reminder, a short message service (SMS) was provided every evening around 9PM. The original German version of the PAQ24 (with a non-validated English translation) is included in the Additional file 1. The English version was translated by the authors for illustration purposes. Due to the lack of a correct translation process we do not recommend using this version.

We made three important changes compared to the IPAQ-SF. First, we changed the recall period to a $24 \mathrm{~h}$ day. Secondly, we included separate questions about resistance training, swimming, cycling for transport and cycling for exercise. The inclusion of resistance training should increase face validity since this is an essential part of current PA guidelines [1, 4]. Questions regarding swimming and cycling were included because they represent common activities and it is doubtful whether they can be appropriately detected by accelerometers [24]. Thirdly, instead of recalling only activities performed in bouts of at least $10 \mathrm{~min}$, the PAQ24 asks participants to recall activities of any duration (i.e., all daily minutes). The questionnaire was pilot tested with 10 volunteers. Results were discussed and changes were made when required.

The questionnaire refers to all domains of PA (work, transport, recreation, sports, household/gardening) and obtains information regarding duration (minutes per day $[\mathrm{min} /$ day $]$ ), frequency (via the daily assessment) and intensity of PA with seven questions: walking, cycling (separate for transport and exercise), moderate activities and vigorous activities (separate for resistance training, swimming and any other vigorous activities). Two additional questions were included to assess both sedentary time (ST) and physical health status (illness, injury, no symptoms). In addition to the summarized score of all seven PA questions (Total PA), we also addressed the agreements of vigorous PA (VPA) and ST. VPA was calculated by summing up cycling for exercise and the three questions about vigorous activities. Cycling for exercise (e.g., racing) was considered as vigorous intensity according to the Compendium of Physical Activities [25] and other empirical investigations showing MET values $\geq 6$ when cycling in higher speeds, even in trained individuals [26]. Although this study targeted the construct PA, results for ST were presented to allow comparisons with other studies. For additional analyses, the scores Total PA excluding walking, Total PA excluding cycling and Total PA excluding swimming were calculated. This was done due to the limited ability of the accelerometer to detect these activities and potential difficulties in the recall of all walking minutes [14, 24]. The scoring protocol of the PAQ24 can be found in the Additional file 1. All scores were expressed as min/day. Control of plausibility was based on the International Physical Activity Questionnaire (IPAQ) guidelines [27].

\section{GPAQ}

The GPAQ was developed by the World Health Organization (WHO) and is a 16-item validated instrument to assess PA in a usual week within three domains (recreation, work, transport) as well as ST [28]. We used the German version of the questionnaire and performed data cleaning and analysis according to the provided analysis guide [29]. The following scores were calculated: Total PA, VPA (derived from all three domains) and ST. All scores were expressed as min/day.

\section{Accelerometer}

Accelerometers are motion sensors which are able to measure acceleration, i.e. the change in velocity of an object over time [30]. The devices are worn on certain parts of the human body (e.g., hip, wrist, ankle) and measure the acceleration of the respective body segments during movement. Although, accelerometry has limitations when measuring activities that are highly static or insufficient captured due to body placement (e.g., wrist-worn while cycling), its recorded data (units of acceleration due to gravity) can be used to derive the frequency, duration and intensity of PA [31, 32].

We used GENEActiv ${ }^{\circ}$ (Activinsights Ltd., Kimbolton, UK) accelerometers for the device-based measurement of participants' PA. This triaxial device is water resistant and has a dynamic range of $\pm 8 \mathrm{~g}$. Participants were instructed to wear the accelerometer, in shape and dimension of a conventional watch, on their non-dominant wrist for $24 \mathrm{~h}$ 
per day for seven consecutive days. In addition, participants recorded any non-wear periods in a diary. Acceleration was recorded at $100 \mathrm{~Hz}$ and raw data was extracted using GENEActiv PC software version 3.2. Signal processing was performed in $\mathrm{R}$ (version 3.5.1; http://cran.r-project.org) using package GGIR (version 1.6-7).

Verification of sensor calibration error was performed and files were considered adequate for analyses if postcalibration error was less than $0.02 \mathrm{~g}[33,34]$. Non-wear was classified for a moving window of $60 \mathrm{~min}$ (with 15 min increments) if the standard deviation was less than 13 milligravity units $(\mathrm{mg})$ or the range of values was less than $50 \mathrm{mg}$ for at least two out of three axes [33, 35]. This window assures that short periods of sleep or inactivity were not misclassified as non-wear [35]. In presence of misclassification, the time stamp of the inactivity periods in addition to the information provided in the diary was used to determine non-wear periods more precisely. The algorithm to detect periods of inactivity was described previously [36]. Each accelerometer file was visually checked by two researchers. The vector magnitude (expressed in $\mathrm{mg}$ ) using the Euclidean norm minus $1 \mathrm{~g}$ (ENMO: $\sqrt{a_{x}^{2}+a_{y}^{2}+a_{z}^{2}}-1 \mathrm{~g}$ ) was calculated based on $5 \mathrm{~s}$ epochs [35]. Any negative values were rounded up to zero. Because most participants started wearing the device in the morning of the first day, we considered 5 $\mathrm{AM}$ as the start of the measurement. For each day, sleep duration was estimated using a heuristic algorithm looking at the Distribution of Change in Z-Angle (HDCZA) [37]. Finally, total waking time was calculated as $24 \mathrm{~h}$ minus sleep duration. A day was considered as valid when waking non-wear time was less than $20 \%$ of the total waking time.

Thresholds for moderate and vigorous PA were considered as $100 \mathrm{mg}$ and $400 \mathrm{mg}$, respectively [38]. Inactivity was defined as any time $<50 \mathrm{mg}$ excluding sleep and non-wear $[38,39]$. Unbouted time spent in different intensity categories was calculated (i.e., based on all $5 \mathrm{~s}$ epochs; expressed in min/day). Variables included in the analyses were Inactivity $(<50 \mathrm{mg})$, Total PA $(\geq 50 \mathrm{mg})$, MVPA ( $\geq 100 \mathrm{mg}$ ), VPA ( $\geq 400 \mathrm{mg}$ ). Relative frequency of time spent in each intensity category based on the time the accelerometer was worn was multiplied by the total waking wear time to obtain non-wear adjusted minutes of PA and Inactivity.

\section{Sample size}

Minimum coefficients for sufficient construct validity have been suggested $[7,8]$. To detect the minimum correlation of 0.4 between scores from the questionnaire and accelerometer with a power of at least $80 \%(\alpha=$ 0.05 , two-tailed), a sample size of $n \geq 46$ was required as calculated using G*Power version 3 [40]. This sample size will also allow sufficient precision in the estimation of the effect (i.e., 95\% confidence interval $[\mathrm{CI}]$ with a maximum width of \pm 0.25 ) [41].

\section{Statistical analysis}

Descriptive statistics are presented using median and interquartile range (IQR) for PA variables and mean and standard deviation for all other continuous variables (unless otherwise stated). The extent of agreement between variables of PAQ24 and accelerometer was assessed for each day (i.e., total minutes of each day) and the overall week (i.e., $\mathrm{min} /$ day as an average across the full measurement period). Daily and weekly scores of PAQ24 (Total PA, VPA, ST) were compared to related variables of GPAQ (Total PA, VPA, ST) and accelerometer (Total PA, MVPA, VPA, Inactivity). Concerning all daily comparisons between PAQ24 and accelerometer, only days with valid data in both instruments were included.

Spearman correlation coefficients $(\rho)$ were used to determine the relative agreement. Weighted Spearman correlation coefficients were applied when there were missing days in either the accelerometer or PAQ24 assessment and weekly scores of the two instruments were addressed. A weighted analysis is recommended in presence of different numbers of repeated observations [42]. Bland-Altman analysis including mean difference and 95\% limits of agreement (LOA) was used to evaluate absolute agreement [43]. Spearman correlations coefficients between weekly absolute differences and the average $\left(\rho_{\text {diff }}\right)$ were reported for Total PA, VPA and ST. Finally, mean differences were expressed as percentage (\%), showing either average over- or under-reporting.

Sensitivity analyses were performed using the additional scores of the PAQ24 to assess whether agreements vary depending on the questionnaire score and accelerometer intensity category. Therefore, the agreement with the accelerometer was assessed for Total PA excluding walking, Total PA excluding cycling and Total PA excluding swimming. These scores as well as Total PA of the PAQ24 were also compared to both Total PA $(\geq 50 \mathrm{mg}$ ) and MVPA ( $\geq 100 \mathrm{mg}$ ) from the accelerometer. Mann-Whitney $U$ tests were used to compare included and non-included participants (e.g., due to missing accelerometer data) regarding sociodemographic characteristics and self-reported PA within the PAQ24. Statistical analyses were performed using SPSS Statistics version 25 (IBM Corp, Armonk, NY, USA) and GraphPad Prism version 7 (GraphPad Software, La Jolla, CA, USA).

\section{Results}

Participants and descriptive data

Of 70 students invited, 50 agreed to participate. All participants were found to be eligible and completed all 
parts of the study. In total, 39 (78\%) men and $11(22 \%)$ women participated (mean age $=25.1 \pm 2.5$, mean $\mathrm{BMI}=$ $23.7 \pm 2.0$ ). Mean aerobic capacity, represented by participants' $\mathrm{VO}_{2 \max }$, was $50.6 \pm 7.1 \mathrm{ml} / \mathrm{kg} / \mathrm{min}$ (female: $44.9 \pm$ 5.7 , male: $52.3 \pm 6.8$ ).

All participants had valid data for all seven days of the PAQ24 measurement period, except for one participant who did not report ST on Monday. Among 350 days of accelerometry, six days were excluded due to technical problems and 24 days due to not meeting the criterion for a valid day. Three of the 50 participants had less than five valid days, whereas four participants had five valid days and 12 participants had six valid days. As a result, a total of 320 days (319 for ST) were included in all analyses comparing PAQ24 and accelerometer. Two participants reported implausible high values in the GPAQ and, thus, 48 (47 for ST) participants were included in all analyses comparing PAQ24 and GPAQ.

Table 1 shows minutes of daily and weekly PA and ST scores of PAQ24 and corresponding measures from the accelerometer. Minutes of all additional scores of the PAQ24 (i.e., Total PA excluding walking [MVPA], Total PA excluding cycling, Total PA excluding swimming) are provided in the Additional file 2. Participants reported a median duration of 1519 min (IQR: 1015-2203) of Total PA over the full measurement period with an average of $234 \pm 97 \mathrm{~min} /$ day. Participants with missing accelerometer data on Saturday $(n=9)$ or Sunday $(n=7)$ were comparable to included participants regarding age, BMI and Total PA of PAQ24 $(p>0.05)$. Median accelerometer waking non-wear was $12 \mathrm{~min} /$ day (IQR: 0-35).

\section{Experiences with the PAQ24}

Some participants reported that the recall of ST and walking time was difficult. Another common answer was the difficulty to report the total amount of active and inactive minutes and to differentiate between different intensities of PA. Participants' recommendations to improve the questionnaire were to provide better explanations for moderate and vigorous PA, more examples and the opportunity to complete the questionnaire more than once per day.

\section{Comparison between questionnaires}

In the GPAQ, participants reported a median duration of $157 \mathrm{~min} /$ day (IQR: 102-240) of Total PA, $51 \mathrm{~min} /$ day (IQR: $30-86$ ) of VPA and $360 \mathrm{~min} /$ day (IQR: 270-480) of ST. Results for relative and absolute agreement between the questionnaires are reported in Table 2. A correlation of $\rho=0.43$ was observed for Total PA. Compared to the GPAQ, the PAQ24 under-reported VPA on average by $20 \%$ and ST by $0.3 \%$ whereas overreported Total PA by $36 \%$.

\section{Comparison with accelerometer}

Daily and weekly relative agreements of Total PA, VPA and ST of PAQ24 with corresponding measures from the accelerometer (Total PA, VPA, Inactivity) are shown in Figs. 1 and 2. Correlation coefficients for daily Total PA ranged from 0.37 to 0.72 with correlations of $\geq 0.50$ on five out of seven days. For the overall week, the highest correlation was observed for Total PA $(\rho=0.44, p=0.002)$.

Figure 3 shows mean differences and 95\% LOA of Total PA for each day. The smallest 95\% LOA were observed on Monday (56 $\pm 222 \mathrm{~min})$. A positive mean difference (i.e., higher accelerometer values) was evident for all days ranging from 37 to $87 \mathrm{~min}$. These mean differences showed that daily Total PA was consistently under-reported by the PAQ24 (range: 12 to 32\%).

Similar 95\% LOA were observed for weekly Total PA (Fig. 4) including a correlation of $\rho_{\text {diff }}=-0.42(95 \%$ CI: $-0.63--0.16 ; p=0.002$ ) between the absolute differences and the average. Compared to the accelerometer, the PAQ24 under-reported weekly Total PA by $21 \%$ on

Table 1 Daily and weekly minutes of PA, ST and Inactivity measures from PAQ24 and accelerometer

\begin{tabular}{|c|c|c|c|c|c|c|c|}
\hline & \multicolumn{3}{|c|}{ PAQ24 } & \multicolumn{4}{|c|}{ ACC } \\
\hline & Total PA & VPA & ST & Total PA & MVPA & VPA & Inactivity \\
\hline Monday & $213(140-289)$ & $45(0-91)$ & $380(240-455)^{a}$ & $296(182-361)$ & $154(98-213)$ & $8(2-28)$ & $622(512-654)^{a}$ \\
\hline Tuesday & $200(148-278)$ & $60(0-85)$ & $360(240-510)$ & $271(214-379)$ & 148 (99-192) & $9(3-23)$ & 685 (599-736) \\
\hline Wednesday & $205(130-340)$ & $0(0-60)$ & $330(240-510)$ & $290(230-366)$ & 159 (115-208) & $13(3-27)$ & $662(605-727)$ \\
\hline Thursday & $168(106-360)$ & $30(0-75)$ & $360(240-472)$ & $292(212-387)$ & $155(101-213)$ & $10(2-26)$ & 679 (579-767) \\
\hline Friday & $220(161-351)$ & $60(0-103)$ & $360(203-555)$ & $295(227-406)$ & $146(120-230)$ & $8(4-16)$ & 667 (558-758) \\
\hline Saturday & $230(160-330)$ & $40(0-90)$ & $270(180-390)$ & $286(236-375)$ & 140 (116-184) & $10(4-21)$ & $662(586-755)$ \\
\hline Sunday & 155 (95-245) & $0(0-70)$ & $330(248-460)$ & $260(198-333)$ & 127 (87-185) & $8(2-21)$ & $693(588-767)$ \\
\hline Average/day & $234 \pm 97$ & $52 \pm 36$ & $366 \pm 137$ & $298 \pm 67$ & $159 \pm 45$ & $15 \pm 9$ & $656 \pm 81$ \\
\hline
\end{tabular}

Notes: All data presented in minutes using median (IQR) or mean \pm SD based on either 46 (Monday), 49 (Tuesday), 47 (Wednesday), 48 (Thursday), 46 (Friday), 43 (Saturday), 41 (Sunday) or 50 (average per day) participants. ACC accelerometer, IQR interquartile range, MVPA moderate-to-vigorous physical activity, PA physical activity, PAQ24 Physical Activity Questionnaire for $24 \mathrm{~h}, S D$ standard deviation, ST sedentary time, VPA vigorous physical activity. ${ }^{a}$ based on 45 participants due to four invalid days in the accelerometer assessment and another missing value in the PAQ24 
Table 2 Relative and absolute agreement of Total PA, VPA and ST between PAQ24 and GPAQ

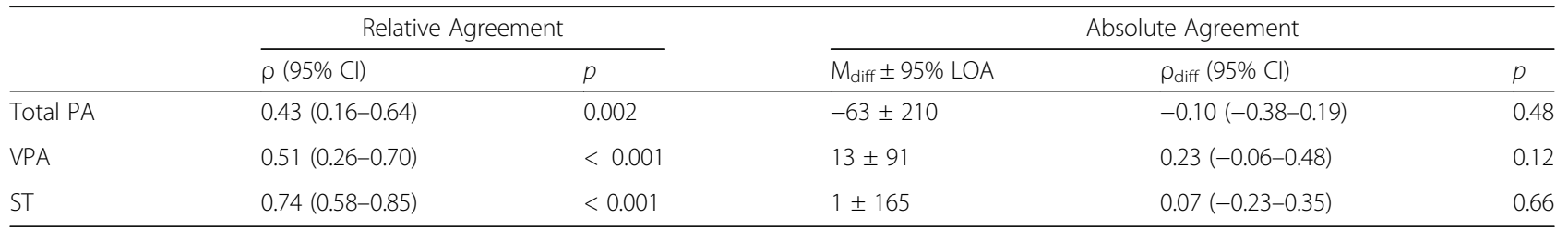

Notes: Results were based on either 48 (Total PA, VPA) or 47 (ST) participants. Relative agreement was assessed by Spearman correlations coefficients ( $\rho$ ) and absolute agreement by Bland-Altman analyses including mean difference and $95 \%$ limits of agreement (reported as minutes per day) and corresponding Spearman correlation coefficients between absolute differences and the average of the two questionnaires ( $\rho_{\text {diff }}$ ). $C I$ confidence interval, GPAQ Global Physical Activity Questionnaire, LOA limits of agreement, PA physical activity, PAQ24 Physical Activity Questionnaire for $24 \mathrm{~h}, S T$ sedentary time, VPA vigorous physical activity

average per day. Results of absolute agreement for daily and weekly VPA and ST are shown in the Additional files 3 and 4 . Absolute agreement was $-36 \pm 70$ $\mathrm{min} /$ day $\quad\left(\rho_{\text {diff }}=-0.85, \quad 95 \% \quad \mathrm{CI}: \quad-0.91 \quad-\quad-0.74\right.$; $p<0.001)$ for weekly VPA and $290 \pm 256 \mathrm{~min} /$ day $\left(\rho_{\text {diff }}=\right.$ $-0.45,95 \%$ CI: $-0.65--0.20 ; p=0.001$ ) for weekly ST. This means that, compared to the accelerometer, the PAQ24 over-reported weekly VPA by $338 \%$ and underreported weekly ST by $44 \%$ on average per day.

Results of all additional analyses are reported in the Additional files 5 and 6 (relative agreement) and Additional file 7 (absolute agreement). These files also show the results for the agreement between PAQ24 and aerobic capacity (e.g., $\rho=-0.07$ for Total PA). Compared to weekly Total PA, similar correlations were observed for weekly Total PA excluding walking $(\rho=0.38)$, weekly

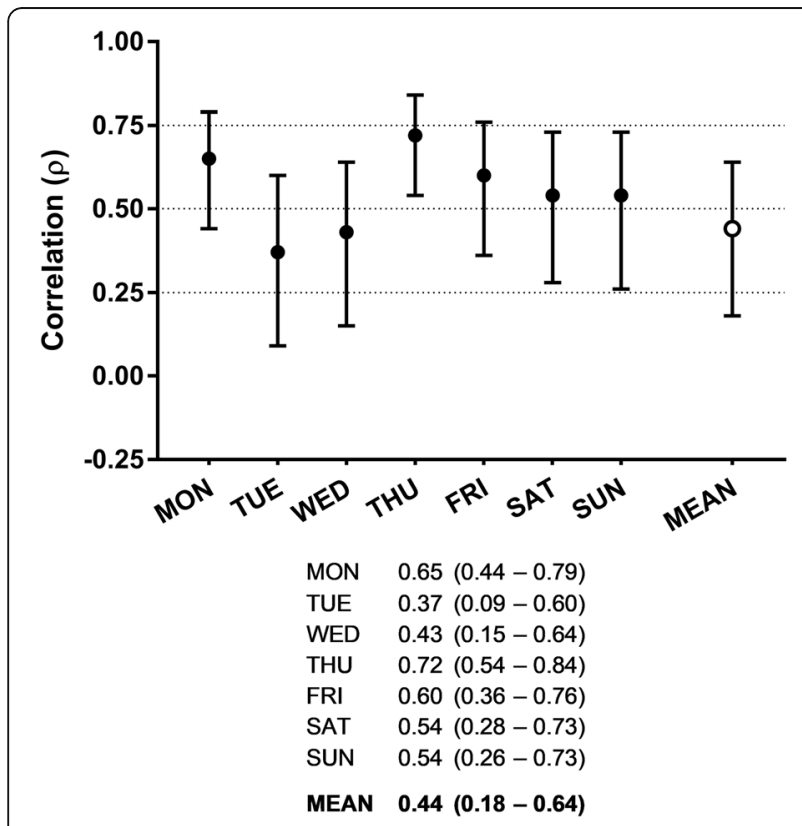

Fig. 1 Summary of daily and weekly relative agreement of Total PA between PAQ24 and accelerometer. Results are presented as Spearman correlation coefficients with 95\% confidence intervals. PA physical activity, PAQ24 Physical Activity Questionnaire for $24 \mathrm{~h}$
Total PA excluding cycling $(\rho=0.41)$ and weekly Total PA excluding swimming $(\rho=0.41)$. Comparing weekly Total PA of the PAQ24 with MVPA instead of Total PA from the accelerometer resulted in a lower relative agreement $(\rho=0.29)$. This was similar, although usually less pronounced, for the additional scores of the PAQ24. When comparing weekly Total PA of PAQ24 with MVPA, instead of Total PA, from the accelerometer, absolute agreements changed. The width of the $95 \%$ LOA was similar but changes in the mean difference were observed $(-75 \pm$ $183 \mathrm{~min} /$ day; $\rho_{\text {diff }}=-0.65,95 \%$ CI: $-0.79-0.46$; $p<0.001)$. Similar results for absolute and relative agreements were obtained for each day.

\section{Discussion}

The purpose of this study was to assess the construct validity of a short self-administered daily PA questionnaire (PAQ24) in young active adults. We expected that the short recall period will result in satisfying construct validity. However, the results of the study revealed inconclusive evidence for the construct validity of the PAQ24. Compared to accelerometry, the PAQ24 showed satisfying relative agreements (i.e., $\rho \geq 0.5$ ) on five out of seven days when assessing Total PA. The relative agreements for the overall week (i.e., averages per day) were unsatisfying for all scores, including Total PA. Similar moderate, but not satisfying $(\rho<0.70)$, agreements were observed when comparing scores of PAQ24 and GPAQ. Furthermore, absolute agreements for both daily and weekly scores were poor because of wide LOA. Additional analyses using different scores of the PAQ24 or accelerometer intensity cut points resulted in similar or lower agreements.

PA reported in the PAQ24 varied from day to day with the highest minutes on Saturday and the lowest on Sunday. This variation may be influenced by daily differences in the amount of leisure time, participation in sport events or convenience of scheduling [44]. For daily PA assessments, it is important to consider this variation in PA [45]. In general, the use of multiple short-term measurements should increase the ability of the instrument to distinguish between true variation in PA and other sources of error [46]. However, depending on the data collection 

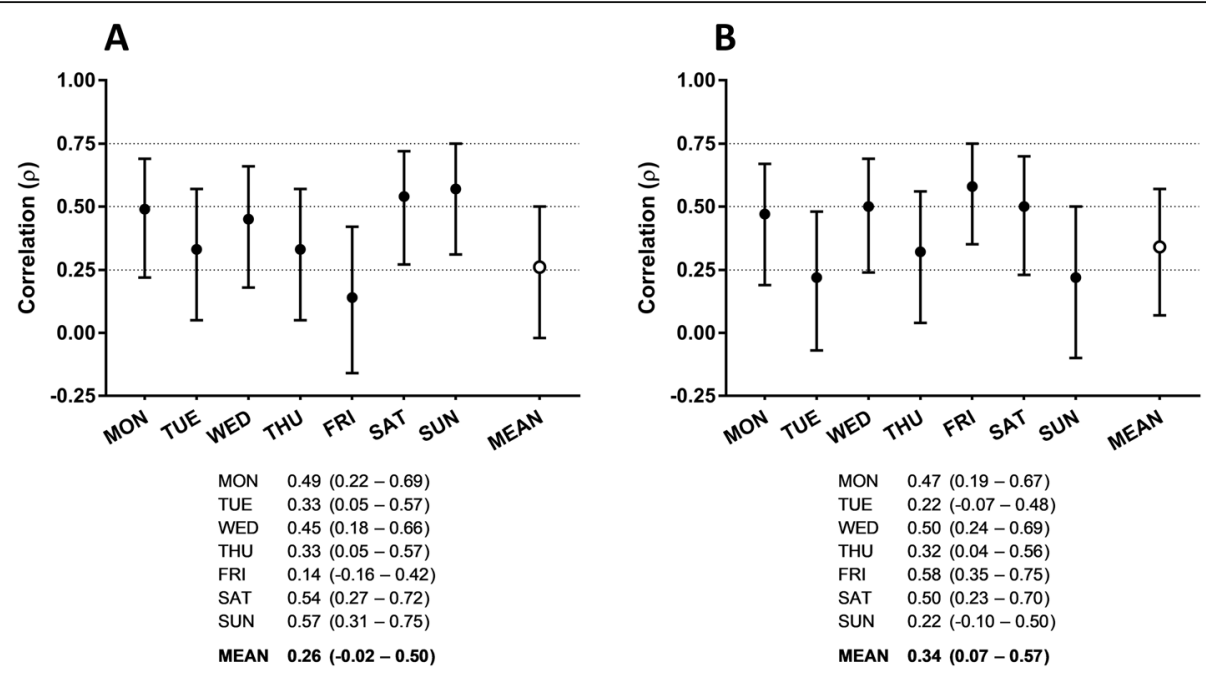

Fig. 2 Summary of daily and weekly relative agreement of VPA (a) and ST (b) between PAQ24 and accelerometer. Results are presented as Spearman correlation coefficients with 95\% confidence intervals. PAQ24 Physical Activity Questionnaire for 24 h, ST sedentary time, VPA vigorous physical activity

method, study population and choice of PA score under investigation, the number of measurements needed to capture this true variation in PA may vary. For example, three to five days of accelerometer monitoring might be needed to obtain accurate levels of PA in adults [45, 47] but more days are required for the assessment of Inactivity [48], when using self-report methods [46], or in specific populations such as children [49]. The impact of the variance in PA behaviors on reliability and the minimum number of measurements when using daily PA questionnaires such as the PAQ24 must be evaluated in future studies. This would also help in drawing conclusions about the feasibility of such a questionnaire.

Overall, the daily results for Total PA were comparable to more sophisticated $24 \mathrm{~h}$ diaries and recalls $[8,11,18$, 50]. The relative agreement of weekly Total PA between PAQ24 and accelerometer did not meet our criterion for satisfying validity but was in the upper range of results when using previous questionnaires $[8,11,51]$. For example, a systematic review of 23 studies on the construct validity of the IPAQ-SF reported correlations ranging from 0.09 to 0.39 for total PA when compared to devicebased measures of PA [51]. Absolute agreement of weekly PA scores of the PAQ24 was rather poor. We observed smaller LOA for weekly compared to daily scores due to reduced random error when using averages of multiple measurements. Moreover, increases in Total PA and VPA were associated with changes in the observed difference between PAQ24 and accelerometer (e.g., shifted from under- to over-reporting with increasing PA scores).

The lack of agreement between PAQ24 and accelerometer may be attributable to differences in individual characteristics and the measurement quality of both methods.
For example, it has been shown that brief, unstructured or low-intensity activities are difficult to recall $[13,14]$ and that the level of agreement varies depending on factors such as age, weight-status or accelerometer data processing [52]. Our participants perceived difficulties with classifying the intensity of activities and with reporting the total volume of walking time. On the other side, acceleration of activities such as cycling or resistance training may not be able to be accurately captured by device-based measurement [24]. However, no improvements in the agreement were observed after excluding these activities. We also observed daily variation in the agreements, which could be influenced by both random and systematic error. For example, activities which are poorly detected by the accelerometer may have been performed on specific days. Likewise, some days may include more structured activities and events (e.g., exercise sessions, competitions) which are easier to recall [13].

Neither questionnaires nor accelerometers are perfect tools to measure PA. This lack of real gold standard was correctly acknowledged by several researchers $[6,53]$. In addition to the disadvantage of reporting errors, a further limitation is, that questionnaires are always developed for a specific population (e.g., elderly, adults, youth, pregnancy) and the identification of most qualified ones is difficult [7, $8,54,55]$. Moreover, the interpretation of questions in the questionnaire (e.g. intensity description) is influenced by characteristics of the participant such as perceived confidence [56] and origin (e.g., different countries and cultures need cross-cultural adaptations) [57]. These individual characteristics can limit the measurement quality and may result in an under- or overestimation of self-reported PA. On the other side, PA data derived from accelerometry is 

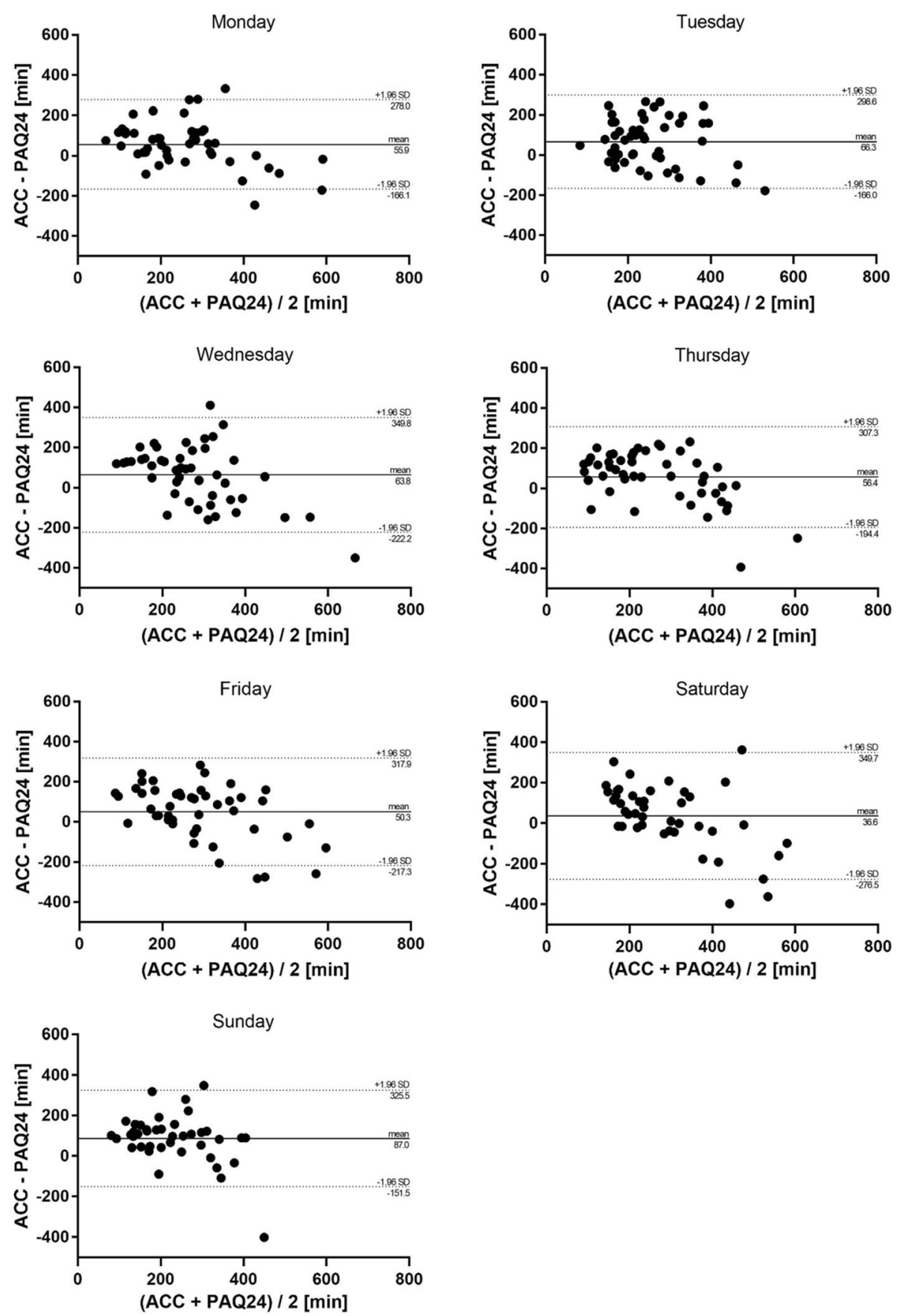

Fig. 3 Absolute agreement of daily Total PA between PAQ24 and accelerometer. Bland-Altman plots for each day showing difference versus average of the values measured by the two methods with 95\% limits of agreement. ACC accelerometer, PA physical activity, PAQ24 Physical Activity Questionnaire for $24 \mathrm{~h}$

influenced by several decisions of the researcher. Depending on brand [58], body placement $[59,60]$ and sampling frequency [47], the data, used for subsequent analyzing, is already affected by researchers' pre-choices. Also, several other decisions (e.g., intensity cut points, epoch length, filters, algorithms to detect non-wear, requirements for a valid day/week) have been shown to influence the PA estimates from the accelerometer $[47,61]$. The current lack of consensus on best practices to handle accelerometer data hampers the quality of the assessment of measurement properties of PA questionnaires (since accelerometers are often considered as "reference" measure).

The agreements for the overall week between PAQ24 and accelerometer were lower than what we would 


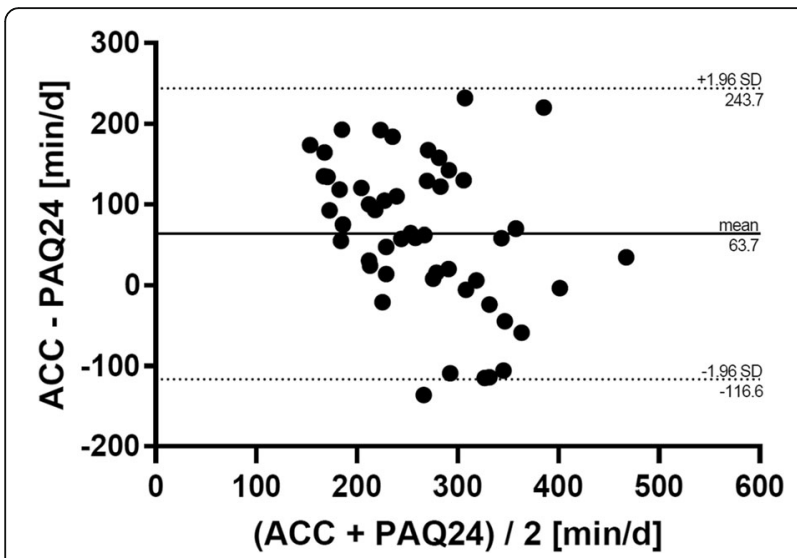

Fig. 4 Absolute agreement of weekly Total PA between PAQ24 and accelerometer. Bland-Altman plots for each day showing difference versus average of the values measured by the two methods with 95\% limits of agreement. ACC accelerometer, PA physical activity, PAQ24 Physical Activity Questionnaire for $24 \mathrm{~h}$

assume based on the pattern in the daily results. This may be due to a stronger influence of different systematic (e.g., additive and multiplicative) errors [62]. For example, consistent under- or over-reporting of PA can influence the estimation of mean, dispersion or participants' ranking order when considering averages per day. Such a reporting bias may only exist for some but not all participants (differential recall bias) and can either increase or decrease the level of agreement [63]. Furthermore, our results showed that the true level of PA was also related to the level of agreement, namely by changes in over- and under-reporting with different PA levels (see results from Bland-Altman analysis). These influences on the repeatability of the tools could have reduced the weekly compared to daily relative agreements, even if one instrument would be free of error [64].

The results also demonstrated poor absolute agreements and only moderate correlations between PAQ24 and GPAQ. Neither Total PA nor VPA did meet our criterion for satisfying construct validity. However, similar correlations were reported in previous studies when comparing forms of the IPAQ with the GPAQ $[65,66]$. This lower agreement can be influenced by differences in the questionnaire format. The PAQ24 includes separate questions for cycling, walking, swimming and resistance training whereas the GPAQ combines these activities into fewer questions and obtains information using different domains of PA [28]. The use of different recall periods (e.g." typical week" in the GPAQ) could also have reduced the level of agreement [67].

ST of the PAQ24 was strongly related to ST of the GPAQ but less to Inactivity from the accelerometer. Also, Bland-Altman analyses indicated poor absolute agreements for daily and weekly ST which seems to be in line with previous results showing usually an under- reporting of ST compared to the accelerometer [52]. This poor agreement may be partly explained by difficulties in reporting ST, as mentioned by some participants, and the lower accuracy of wrist-worn accelerometers (without further use of inclinometers) to differentiate between non-movement positions such as lying, sitting or standing [68]. However, the results of weekly ST are comparable to previous questionnaires [11]. Finally, PA was not associated with aerobic capacity (see Additional file 5) which may be due to the non-overlapping parts of the two concepts [69], the variability in PA [46] and the homogeneity of the sample regarding their usually high fitness levels.

The results of the present study must be interpreted with respect to our specific sample, since participants involved were highly active and trained students. The participants were affine to sports and exercise, and therefore, should be able to better estimate the intensity of PA compared to a sample with a different background. Many participants were members of a sports club with settled weekdays of training and were registered for obligatory university exercise courses. Taking this into account, it might have been easier for them to recall PA, compared to the general population. This strongly limits the generalizability of our results. Future studies are therefore needed to evaluate the PAQ24 and other promising daily PA questionnaires in representative samples of the general population.

Finally, we tried to improve the measurement properties of PA questionnaires by using a short recall period. Although we modified an existing questionnaire for our purposes, we do not recommend using the PAQ24 to measure PA in other studies. Already in 2000, Sallis and Saelens [5] recognized the existence of too many different questionnaires and recommended to use only the most qualified ones for future research. Therefore, we strongly recommend using an existing questionnaire whenever possible. The choice of the questionnaire should follow the purpose of the study and the evaluation of measurement properties (e.g., content validity, reliability, construct validity, responsiveness). Several reviews on measurement properties of PA questionnaires have been published $[7,8$, $11,53,70]$ and may help in the selection of most qualified questionnaires. However, we invite researchers to use our questionnaire in future validation studies to further improve the measurement quality of PA questionnaires. For example, using smartphone applications for the daily assessment may increase feasibility. Future studies should also evaluate the measurement errors associated with multiple measurements as well as minimum required days of monitoring. Overall, we, together with others [14], argue that multiple short-term recalls are a promising approach to overcome important short-comings of traditional PA questionnaires. 


\section{Strengths and limitations}

First, the specific study population (young active adults) limits the generalizability of the findings. Secondly, although participants were instructed to complete the PAQ24 before going to bed, we did not assess whether they were still awake and active after they completed the questionnaire. Thirdly, results for absolute agreement showed a strong dependence on accelerometer intensity cut points which should be considered when interpreting the results. This seems reasonable when using lower or higher cut points and was rather affecting the mean difference than the magnitude and variation of differences (i.e., LOA). Finally, this study did not assess the effect of a short recall period using an experimental design comparing it with a recall period of a week. On the other side, the study has several strengths: i) the use of raw accelerometry to increase transparency and comparability between studies; ii) reporting the influence of different accelerometer intensity cut points on the results; iii) the use of guidelines regarding the validation of PA questionnaires (e.g., specifying a priori hypotheses) [7, 8, 71]; and iv) data collection was performed within a short period, which reduces the influences caused by changes in weather, seasons or types of activities.

\section{Conclusions}

A short recall period may improve the measurement quality of PA questionnaires as this was seen in the daily results for Total PA (five out of seven days showed sufficient construct validity). In contrast, the weekly results for Total PA did not meet our criterion. Also, agreements with the GPAQ were unsatisfying and absolute agreements were poor. Since the results of the present study were based on a very specific sample, studies in representative samples of the general adult population are needed. Also, the feasibility of a short daily PA questionnaire in large studies as well as the influence of measurement errors should be evaluated in future studies. Altogether, the results of the PAQ24 are promising and researchers are invited to use our questionnaire in future studies exclusively for the purpose of improving the measurement quality of PA questionnaires (e.g., to use smartphone applications for the daily assessment).

\section{Supplementary information}

\section{Supplementary information accompanies this paper at https://doi.org/10.} 1186/s12889-020-8165-3

\section{Additional file 1. German version (original) and translated English} version (not validated) of the PAQ24.

Additional file 2. Daily and weekly minutes of all additional scores of the PAQ24.

Additional file 3. Absolute agreement of daily and weekly VPA between PAQ24 and accelerometer. Bland-Altman plots for each day showing difference versus average of the values measured by the two methods with $95 \%$ limits of agreement.
Additional file 4. Absolute agreement of daily and weekly ST between PAQ24 and accelerometer. Bland-Altman plots for each day showing difference versus average of the values measured by the two methods with 95\% limits of agreement.

Additional file 5. Relative agreement of Total PA, Total PA excl. Walking (MVPA), Total PA excl. Cycling and Total PA excl. Swimming between PAQ24 and different measures of the accelerometer.

Additional file 6. Relative agreement of VPA and ST between PAQ24 and accelerometer.

Additional file 7. Absolute agreement of Total PA, Total PA excl. Walking (MVPA), Total PA excl. Cycling and Total PA excl. Swimming between PAQ24 and different measures of the accelerometer.

\section{Abbreviations}

ACC: Accelerometer; ACT24: Activities completed over time in $24 \mathrm{~h}$; BMI: Body mass index; ENMO: Euclidean norm minus $1 \mathrm{~g}$; GPAQ: Global Physical Activity Questionnaire; HDCZA: Heuristic algorithm looking at distribution of change in Z-Angle; IPAQ: International Physical Activity Questionnaire; IPAQ-SF: International Physical Activity Questionnaire - Short Form; LOA: Limits of agreement; MET: Metabolic equivalent; mg: Milligravity units; MVPA: Moderate-to-vigorous physical activity; PA: Physical activity; PAQ24: Physical Activity Questionnaire for 24 h; SMS: Short message service; ST: Sedentary time; VO2max: Maximum oxygen consumption; VPA: Vigorous physical activity; WHO: World Health Organization

\section{Acknowledgements}

We would like to thank Dominik Hofer, Patrick Ladler, Matthias Schützenhöfer and Nikolai Starman for helping us with the acquisition and entry of data.

Authors' contributions

$J$ J, MCS, MvP, PH and WR, were involved in the design of the study. All assessments were led by $\mathrm{BN}$ and $\mathrm{PH}$. All authors participated in visual inspection of raw data, data preparation, data analysis and the development of the PAQ24. BN, MCS and PH wrote the first draft of the manuscript with input from the other authors. All authors read and approved the final manuscript.

\section{Funding}

None.

Availability of data and materials

The datasets used and/or analyzed during the current study are available from the corresponding author on reasonable request.

\section{Ethics approval and consent to participate}

Ethical approval was obtained by the Ethics Committee of the University of Graz (GZ. 39/51/63 ex 2017/18). Written informed consent was obtained from all participants.

Consent for publication

Not applicable.

\section{Competing interests}

The authors declare that they have no competing interests.

\section{Author details}

${ }^{1}$ Institute of Sport Science, University of Graz, Graz, Austria. ${ }^{2}$ Department of Public Health, Institute for Health Promotion and Disease Prevention (IfGP)، Graz, Austria. ${ }^{3}$ Institute of Sport Science, German University of Health and Sports, Berlin, Germany.

Received: 12 June 2019 Accepted: 7 January 2020

Published online: 16 January 2020

References

1. 2018 Physical Activity Guidelines Advisory Committee. 2018 physical activity guidelines advisory committee scientific report. Washington, DC: US Department of Health and Human Services; 2018. 
2. Rosenbaum S, Tiedemann A, Sherrington C, Curtis J, Ward PB. Physical activity interventions for people with mental illness: a systematic review and meta-analysis. J Clin Psychiatry. 2014;75:964-74. https://doi.org/10.4088/JCP. $13 \mathrm{r} 08765$

3. Lee I-M, Shiroma EJ, Lobelo F, Puska P, Blair SN, Katzmarzyk PT. Effect of physical inactivity on major non-communicable diseases worldwide: an analysis of burden of disease and life expectancy. Lancet. 2012;380:219-29. https://doi.org/10.1016/S0140-6736(12)61031-9.

4. US Department of Health and Human Services. Physical activity guidelines for Americans 2nd ed. Wasington, DC: US Department of Health and Human Services; 2018.

5. Sallis JF, Saelens BE. Assessment of physical activity by self-report: status, limitations, and future directions. Res Q Exerc Sport. 2000;71(Suppl 2):1-14 https://doi.org/10.1080/02701367.2000.11082780

6. van Hees $\mathrm{V}$. The challenge of assessing physical activity in populations Lancet. 2012;380:1555. https://doi.org/10.1016/S0140-6736(12)61876-5

7. Sattler MC, Jaunig J, Watson ED, van Poppel MNM, Mokkink LB, Terwee CB, Dietz P. Physical activity questionnaires for pregnancy: a systematic review of measurement properties. Sports Med. 2018;48:2317-46. https://doi.org/10. 1007/s40279-018-0961-x

8. van Poppel MNM, Chinapaw MJM, Mokkink LB, van Mechelen W, Terwee CB. Physical activity questionnaires for adults: a systematic review of measurement properties. Sports Med. 2010;40:565-600. https://doi.org/10. 2165/11531930-000000000-00000

9. Skender S, Ose J, Chang-Claude J, Paskow M, Brühmann B, Siegel EM, et al. Accelerometry and physical activity questionnaires - a systematic review. BMC Public Health. 2016;16:515. https://doi.org/10.1186/s12889-016-3172-0

10. Goodwin LD, Leech NL. Understanding correlation: factors that affect the size of r. J Exp Educ. 2006;74:249-66. https://doi.org/10.3200/JEXE.74.3.249-266

11. Helmerhorst HJF, Brage S, Warren J, Besson H, Ekelund U. A systematic review of reliability and objective criterion-related validity of physical activity questionnaires. Int J Behav Nutr Phys Act. 2012;9:103. https://doi.org/10. 1186/1479-5868-9-103

12. Herbolsheimer F, Riepe MW, Peter R. Cognitive function and the agreement between self-reported and accelerometer-accessed physical activity. BMC Geriatr. 2018;18:56. https://doi.org/10.1186/s12877-018-0747-x .

13. Shephard RJ. Limits to the measurement of habitual physical activity by questionnaires. Br J Sports Med. 2003:37:197-206. https://doi.org/10.1136/ bjsm.37.3.197

14. Matthews CE, Moore SC, George SM, Sampson J, Bowles HR. Improving selfreports of active and sedentary behaviors in large epidemiologic studies. Exerc Sport Sci Rev. 2012;40:118-26. https://doi.org/10.1097/JES. Ob013e31825b34a0

15. Füzéki $E$, Engeroff $T$, Banzer W. Health benefits of light-intensity physical activity: a systematic review of accelerometer data of the National Health and nutrition examination survey (NHANES). Sports Med. 2017;47:1769-93. https://doi.org/10.1007/s40279-017-0724-0 .

16. Chastin SFM, de Craemer M, de Cocker K, Powell L, van Cauwenberg J, Dall $P$, et al. How does light-intensity physical activity associate with adult cardiometabolic health and mortality? Systematic review with meta-analysis of experimental and observational studies. Br J Sports Med. 2019;53:370-6. https://doi.org/10.1136/bjsports-2017-097563 .

17. Poitras VJ, Gray CE, Borghese MM, Carson V, Chaput J-P, Janssen I, et al. Systematic review of the relationships between objectively measured physical activity and health indicators in school-aged children and youth. Appl Physiol Nutr Metab. 2016;41:S197-239. https://doi.org/10.1139/apnm2015-0663.

18. Matthews CE, Kozey Keadle S, Moore SC, Schoeller DS, Carroll RJ, Troiano RP, Sampson JN. Measurement of active and sedentary behavior in context of large epidemiologic studies. Med Sci Sports Exerc. 2018;50:266-76. https:// doi.org/10.1249/MSS.0000000000001428.

19. Wollmerstedt $\mathrm{N}$, Nöth U, Ince A, Ackermann $\mathrm{H}$, Martell JM, Hendrich C. The Daily Activity Questionnaire: a novel questionnaire to assess patient activity after total hip arthroplasty. J Arthroplasty. 2010;25:475-80.e1-3. https://doi. org/10.1016/j.arth.2009.01.005 .

20. Matthiessen J, Biltoft-Jensen A, Rasmussen LB, Hels O, Fagt S, Groth MV. Comparison of the Danish physical activity questionnaire with a validated position and motion instrument. Eur J Epidemiol. 2008;23:311-22. https:// doi.org/10.1007/s10654-008-9228-4

21. Sykes K. Capacity assessment in the workplace: a new step test. Occup Health. 1995:47:20-2
22. Sykes K, Roberts A. The Chester step test-a simple yet effective tool for the prediction of aerobic capacity. Physiotherapy. 2004;90:183-8. https://doi.org/ 10.1016/j.physio.2004.03.008

23. Craig CL, Marshall AL, Sjorstrom M, Bauman AE, Booth ML, Ainsworth BE, et al. International physical activity questionnaire: 12-country reliability and validity. Med Sci Sports Exerc. 2003;35:1381-95. https://doi.org/10.1249/01. MSS.0000078924.61453.FB

24. Chen KY, Bassett DR. The technology of accelerometry-based activity monitors: current and future. Med Sci Sports Exerc. 2005;37(11):S490-500. https://doi.org/10.1249/01.mss.0000185571.49104.82

25. Ainsworth BE, Haskell WL, Herrmann SD, Meckes N, Bassett DR, Tudor-Locke C, et al. 2011 compendium of physical activities: a second update of codes and MET values. Med Sci Sports Exerc. 2011;43:1575-81. https://doi.org/10. 1249/MSS.0b013e31821ece12.

26. Louis J, Brisswalter J, Morio C, Barla C, Temprado J-J. The electrically assisted bicycle: an alternative way to promote physical activity. Am J Phys Med Rehabil. 2012;91:931-40. https://doi.org/10.1097/PHM.0b013e318269d9bb .

27. IPAQ Research Committee. Guidelines for data processing and analysis of the International Physical Activity Questionnaire (IPAQ)-short and long forms; 2005.

28. Armstrong T, Bull F. Development of the World Health Organization global physical activity questionnaire (GPAQ). J Public Health. 2006;14:66-70. https://doi.org/10.1007/s10389-006-0024-x .

29. World Health Organization. Global Physical Activity Surveillance. 2018. https:// www.who.int/ncds/surveillance/steps/GPAQ/en/. Accessed 13 Jan 2018.

30. Hills AP, Mokhtar N, Byrne NM. Assessment of physical activity and energy expenditure: an overview of objective measures. Front Nutr. 2014;1:5. https://doi.org/10.3389/fnut.2014.00005

31. Strath SJ, Kaminsky LA, Ainsworth BE, Ekelund U, Freedson PS, Gary RA, et al. Guide to the assessment of physical activity: clinical and research applications: a scientific statement from the American Heart Association. Circulation. 2013; 128:2259-79. https://doi.org/10.1161/01.cir.0000435708.67487.da .

32. Matthews CE. Calibration of accelerometer output for adults. Med Sci Sports Exerc. 2005;37(11):S512-22. https://doi.org/10.1249/01.mss.0000185659.11982.3d

33. da Silva IC, van Hees VT, Ramires W, Knuth AG, Bielemann RM, Ekelund U, et al. Physical activity levels in three Brazilian birth cohorts as assessed with raw triaxial wrist accelerometry. Int J Epidemiol. 2014;43:1959-68. https://doi. org/10.1093/ije/dyu203.

34. van Hees VT, Fang Z, Langford J, Assah F, Mohammad A, da Silva ICM, et al. Autocalibration of accelerometer data for free-living physical activity assessment using local gravity and temperature: an evaluation on four continents. J Appl Physiol. 2014;117:738-44. https://doi.org/10.1152/ japplphysiol.00421.2014

35. van Hees VT, Gorzelniak L, Dean León EC, Eder M, Pias M, Taherian S, et al. Separating movement and gravity components in an acceleration signal and implications for the assessment of human daily physical activity. PLoS One. 2013:8:e61691. https://doi.org/10.1371/journal.pone.0061691.

36. van Hees VT, Sabia S, Anderson KN, Denton SJ, Oliver J, Catt M, et al. A novel, open access method to assess sleep duration using a wrist-worn accelerometer. PLoS One. 2015;10:e0142533. https://doi.org/10.1371/journal. pone.0142533 .

37. van Hees VT, Sabia S, Jones SE, Wood AR, Anderson KN, Kivimäki M, et al. Estimating sleep parameters using an accelerometer without sleep diary. Sci Rep. 2018:8:12975. https://doi.org/10.1038/s41598-018-31266-z

38. Hildebrand $M$, van Hees VT, Hansen $B H$, Ekelund U. Age group comparability of raw accelerometer output from wrist- and hip-worn monitors. Med Sci Sports Exerc. 2014;46:1816-24. https://doi.org/10.1249/ MSS.0000000000000289

39. Horta BL, Schaan BD, Bielemann RM, Vianna CÁ, Gigante DP, Barros FC, et al. Objectively measured physical activity and sedentary-time are associated with arterial stiffness in Brazilian young adults. Atherosclerosis. 2015;243: 148-54. https://doi.org/10.1016/j.atherosclerosis.2015.09.005 .

40. Faul F, Erdfelder E, Buchner A, Lang A-G. Statistical power analyses using G*power 3.1: tests for correlation and regression analyses. Behav Res Methods. 2009;41:1149-60. https://doi.org/10.3758/BRM.41.4.1149

41. Moinester M, Gottfried R. Sample size estimation for correlations with prespecified confidence interval. TQMP. 2014;10:124-30. https://doi.org/10. 20982/tamp.10.2.p0124

42. Bland JM, Altman DG. Statistics notes: calculating correlation coefficients with repeated observations: part 2--correlation between subjects. BMJ. 1995; 310:633. https://doi.org/10.1136/bmj.310.6980.633 
43. Bland JM, Altman DG. Statistical methods for assessing agreement between two methods of clinical measurement. Lancet. 1986;1:307-10.

44. Drenowatz C, Gribben N, Wirth MD, Hand GA, Shook RP, Burgess S, Blair SN. The association of physical activity during weekdays and weekend with body composition in young adults. J Obes. 2016;2016:8236439. https://doi. org/10.1155/2016/8236439

45. Baranowski T, Mâsse LC, Ragan B, Welk G. How many days was that? We're still not sure, but we're asking the question better! Med Sci Sports Exerc. 2008;40(7):S544-9. https://doi.org/10.1249/MSS.0b013e31817c6651 .

46. Matthews CE, Hebert JR, Freedson PS, Stanek EJ, Merriam PA, Ebbeling CB, Ockene IS. Sources of variance in daily physical activity levels in the seasonal variation of blood cholesterol study. Am J Epidemiol. 2001;153: 987-95.

47. Migueles JH, Cadenas-Sanchez C, Ekelund U, Delisle Nyström C, MoraGonzalez J, Löf M, et al. Accelerometer data collection and processing criteria to assess physical activity and other outcomes: a systematic review and practical considerations. Sports Med. 2017;47:1821-45. https://doi.org/ 10.1007/s40279-017-0716-0.

48. Matthews CE, Ainsworth BE, Thompson RW, Bassett DR. Sources of variance in daily physical activity levels as measured by an accelerometer. Med Sci Sports Exerc. 2002;34:1376-81. https:/doi.org/10.1097/00005768-200208000-00021 .

49. Trost SG, Pate RR, Freedson PS, Sallis JF, Taylor WC. Using objective physical activity measures with youth: how many days of monitoring are needed? Med Sci Sports Exerc. 2000;32:426-31.

50. Kohler S, Behrens G, Olden M, Baumeister SE, Horsch A, Fischer B, Leitzmann MF. Design and evaluation of a computer-based 24-hour physical activity recall (cpar24) instrument. J Med Internet Res. 2017;19:e186. https://doi.org/ 10.2196/jmir.7620

51. Lee PH, Macfarlane DJ, Lam TH, Stewart SM. Validity of the International Physical Activity Questionnaire Short Form (IPAQ-SF): a systematic review. Int J Behav Nutr Phys Act. 2011;8:115. https://doi.org/10.1186/1479-5868-8-115 .

52. Cerin E, Cain KL, Oyeyemi AL, Owen N, Conway TL, Cochrane T, et al. Correlates of agreement between accelerometry and self-reported physical activity. Med Sci Sports Exerc. 2016;48:1075-84. https://doi.org/10.1249/MSS. 0000000000000870

53. Dowd KP, Szeklicki R, Minetto MA, Murphy MH, Polito A, Ghigo E, et al. A systematic literature review of reviews on techniques for physical activity measurement in adults: a DEDIPAC study. Int J Behav Nutr Phys Act. 2018; 15:15. https://doi.org/10.1186/s12966-017-0636-2

54. Forsén L, Loland NW, Vuillemin A, Chinapaw MJM, van Poppel MNM, Mokkink LB, et al. Self-administered physical activity questionnaires for the elderly. Sports Med. 2010;40:601-23. https://doi.org/10.2165/11531350000000000-00000

55. Hidding LM, Chinapaw MJM, van Poppel MNM, Mokkink LB, Altenburg TM. An updated systematic review of childhood physical activity questionnaires. Sports Med. 2018;48:2797-842. https://doi.org/10.1007/s40279-018-0987-0 .

56. Cust AE, Armstrong BK, Smith BJ, Chau J, van der Ploeg HP, Bauman A. Selfreported confidence in recall as a predictor of validity and repeatability of physical activity questionnaire data. Epidemiology. 2009;20:433-41. https:// doi.org/10.1097/EDE.0b013e3181931539

57. Loyen A, van Hecke L, Verloigne M, Hendriksen I, Lakerveld J, SteeneJohannessen J, et al. Variation in population levels of physical activity in European adults according to cross-European studies: a systematic literature review within DEDIPAC. Int J Behav Nutr Phys Act. 2016;13:72. https://doi. org/10.1186/s12966-016-0398-2 .

58. Rowlands AV, Yates T, Davies M, Khunti K, Edwardson CL. Raw accelerometer data analysis with GGIR R-package: does accelerometer brand matter? Med Sci Sports Exerc. 2016;48:1935-41. https://doi.org/10. 1249/MSS.0000000000000978.

59. Esliger DW, Tremblay MS. Technical reliability assessment of three accelerometer models in a mechanical setup. Med Sci Sports Exerc. 2006;38: 2173-81. https://doi.org/10.1249/01.mss.0000239394.55461.08

60. Trost SG, Zheng Y, Wong W-K. Machine learning for activity recognition: hip versus wrist data. Physiol Meas. 2014;35:2183-9. https://doi.org/10.1088/ 0967-3334/35/11/2183

61. Orme M, Wijndaele K, Sharp SJ, Westgate K, Ekelund U, Brage S. Combined influence of epoch length, cut-point and bout duration on accelerometryderived physical activity. Int J Behav Nutr Phys Act. 2014;11:34. https://doi. org/10.1186/1479-5868-11-34.

62. Bennett DA, Landry D, Little J, Minelli C. Systematic review of statistical approaches to quantify, or correct for, measurement error in a continuous exposure in nutritional epidemiology. BMC Med Res Methodol. 2017;17:146. https://doi.org/10.1186/s12874-017-0421-6 .

63. Neugebauer R, Ng S. Differential recall as a source of bias in epidemiologic research. J Clin Epidemiol. 1990;43:1337-41.

64. Bland JM, Altman DG. Measuring agreement in method comparison studies. Stat Methods Med Res. 1999:8:135-60. https://doi.org/10.1177/ 096228029900800204

65. Bull FC, Maslin TS, Armstrong T. Global physical activity questionnaire (GPAQ): nine country reliability and validity study. J Phys Act Health. 2009;6: 790-804. https://doi.org/10.1123/jpah.6.6.790

66. Rivière F, Widad FZ, Speyer E, Erpelding M-L, Escalon H, Vuillemin A Reliability and validity of the French version of the global physical activity questionnaire. J Sport Health Sci. 2018;7:339-45. https://doi.org/10.1016/j. jshs.2016.08.004

67. Doma K, Speyer R, Leicht AS, Cordier R. Comparison of psychometric properties between usual-week and past-week self-reported physical activity questionnaires: a systematic review. Int J Behav Nutr Phys Act. 2017;14:10. https://doi.org/10.1186/s12966-017-0470-6 .

68. Rosenberger ME, Haskell WL, Albinali F, Mota S, Nawyn J, Intille S. Estimating activity and sedentary behavior from an accelerometer on the hip or wrist. Med Sci Sports Exerc. 2013;45:964-75. https://doi.org/10.1249/MSS. Ob013e31827f0d9c

69. DeFina LF, Haskell WL, Willis BL, Barlow CE, Finley CE, Levine BD, Cooper KH. Physical activity versus cardiorespiratory fitness: two (partly) distinct components of cardiovascular health? Prog Cardiovasc Dis. 2015;57:324-9. https://doi.org/10.1016/j.pcad.2014.09.008.

70. Silsbury Z, Goldsmith R, Rushton A. Systematic review of the measurement properties of self-report physical activity questionnaires in healthy adult populations. BMJ Open. 2015;5:e008430. https://doi.org/10.1136/bmjopen2015-008430

71. Terwee CB, Mokkink LB, van Poppel MNM, Chinapaw MJM, van Mechelen W, de Vet HCW. Qualitative attributes and measurement properties of physical activity questionnaires: a checklist. Sports Med. 2010;40:525-37. https://doi.org/10.2165/11531370-000000000-00000

\section{Publisher's Note}

Springer Nature remains neutral with regard to jurisdictional claims in published maps and institutional affiliations.
Ready to submit your research? Choose BMC and benefit from:

- fast, convenient online submission

- thorough peer review by experienced researchers in your field

- rapid publication on acceptance

- support for research data, including large and complex data types

- gold Open Access which fosters wider collaboration and increased citations

- maximum visibility for your research: over $100 \mathrm{M}$ website views per year

At $\mathrm{BMC}$, research is always in progress.

Learn more biomedcentral.com/submissions 